\title{
On the multiwavelength spectrum of the microquasar 1E 1740.7-2942 (Research Note)
}

\author{
V. Bosch-Ramon ${ }^{1}$, G. E. Romero ${ }^{2,3}$, J. M. Paredes ${ }^{1}$, A. Bazzano ${ }^{4}$, M. Del Santo ${ }^{4}$, and L. Bassani ${ }^{5}$ \\ 1 Departament d'Astronomia i Meteorologia, Universitat de Barcelona, Av. Diagonal 647, 08028 Barcelona, Catalonia, Spain \\ e-mail: vbosch@am.ub.es; e-mail: jmparedes@ub.edu \\ 2 Instituto Argentino de Radioastronomía, C.C.5, (1894) Villa Elisa, Buenos Aires, Argentina \\ e-mail: romero@iar.unlp.edu.ar \\ Facultad de Ciencias Astronómicas y Geofísicas, UNLP, Paseo del Bosque, 1900 La Plata, Argentina \\ 4 INAF - Istituto di Astrofisica Spaziale e Fisica cosmica di Roma, via del Fosso del Cavaliere 100, 00133 Roma, Italy \\ 5 INAF - Istituto di Astrofisica Spaziale e Fisica cosmica di Bologna, via Gobetti 101, 40129 Bologna, Italy \\ Received 20 April 2006 / Accepted 24 July 2006
}

\section{ABSTRACT}

\begin{abstract}
Context. The microquasar 1E 1740.7-2942 is a source located in the direction of the Galactic Center. It has been detected at X-rays, soft gamma-rays, and in the radio band, showing an extended radio component in the form of a double-sided jet. Although no optical counterpart has been found so far for 1E 1740.7-2942, its X-ray activity strongly points to a galactic nature.

Aims. We aim to improve our understanding of the hard X-ray and gamma-ray production in the system, exploring whether the jet can emit significantly at high energies under the light of the present knowledge.

Methods. We have modeled the source emission, from radio to gamma-rays, with a cold-matter dominated jet model. INTEGRAL data combined with radio and RXTE data, as well as EGRET and HESS upper-limits, are used to compare the computed and the observed spectra.

Results. From our modeling, we find out that jet emission cannot explain the high fluxes observed at hard X-rays without violating at the same time the constraints from the radio data, favoring the corona origin of the hard X-rays. Also, 1E 1740.7-2942 might be detected by GLAST or AGILE at GeV energies, and by HESS and HESS-II beyond $100 \mathrm{GeV}$, with the spectral shape likely affected by photon-photon absorption in the disk and corona photon fields.
\end{abstract}

Key words. X-rays: binaries - stars: winds, outflows - gamma rays: observations - stars: individual: 1E 1740.7-2942 gamma rays: theory

\section{Introduction}

1E 1740.7-2942, discovered at X-rays by the Einstein satellite (Hertz \& Grindlay 1984) and located at less than one degree from the Galactic Center, is considered to be the first microquasar since the observation of radio jets extending $\sim 1^{\prime}$ (Mirabel et al. 1992). There are hints of correlation between the radio and the strong X-ray emission (Mirabel et al. 1993), and X-ray state changes have been observed with timescales similar to those of other X-ray binaries (Del Santo et al. 2005, and references therein). Moreover, a 12.7-days periodical modulation of a 3-4\% in the X-ray emission seems to be produced by the orbital motion of the system (Smith et al. 2002). This X-ray behavior suggests that the source is galactic in nature. The modulation period and the upper-limits in magnitude obtained in the infrared band suggest that the stellar companion in 1E 1740.7-2942 might be a low-mass star (Martí et al. 2000). The behavior of the X-ray spectrum, very similar to those of other galactic black-hole candidates (or BHC) (e.g. Sunyaev et al. 1991), points to a blackhole as the compact object. The inferred star mass, the strong $\mathrm{X}$-ray radiation and the little $\mathrm{X}$-ray orbital modulation, hinting perhaps to a circular orbit, point to accretion via Roche-lobe overflow. The fact that the companion has not been detected yet is likely due to the dense surrounding medium, with high concentrations of dust and a large hydrogen column density of about
$10^{23} \mathrm{~cm}^{-2}$ (Gallo \& Fender 2002), which depletes photons from the infrared up to soft X-ray energies. The same authors also detected hints of an extended X-ray halo. This hydrogen column density is even higher than the typical value in the direction of the Galactic Center ( $\sim 10^{22} \mathrm{~cm}^{-2}$; Dickey \& Lockman 1990), and might imply that this object is embedded within a molecular cloud (Yan \& Dalgarno 1997). This peculiar location could also explain the very extended structures detected at different wavelengths around this source, as was pointed out by Mirabel et al. (1992). Regarding the distance to 1E 1740.7-2942, we have adopted in this work the Galactic Center distance, $8 \mathrm{kpc}$.

Concerning the emission properties, the source shows spectrally flat and faint radio emission coming from the core with flux densities of less than $1 \mathrm{mJy}$ (Fender 2001, and references therein; $\sim 10^{29} \mathrm{erg} \mathrm{s}^{-1}$ at $8 \mathrm{kpc}$ ), as well as optically thin radio emission coming from the radio lobes. In the $10-100 \mathrm{keV}$ band, RXTE and INTEGRAL observed the source in August 2003 when it was in the low-hard state finding fluxes of about $10^{-9} \mathrm{erg} \mathrm{s}^{-1} \mathrm{~cm}^{-2}\left(\sim 10^{37} \mathrm{erg} \mathrm{s}^{-1}\right.$; Del Santo et al. 2005). Further data have been taken by INTEGRAL, which altogether with the previous ones have been reduced and analyzed for this work with the most recent software package (OSA 5.1), obtaining very similar results to those already published. EGRET observed intensively the Galactic Center region in the range $\sim 0.1-10 \mathrm{GeV}$. No source was detected at the position of 1E 1740.7-2942, which 
provides upper-limits for its emission at these energies. At the Galactic Center distance the inferred luminosity upper-limits are about $10^{35} \mathrm{erg} \mathrm{s}^{-1}$ (Hartman et al. 1999). Moreover, the flux of the nearby source 3EG J1744-3011 can be taken as an absolute upper-limit ${ }^{1}$, being about $10^{-10} \mathrm{erg} \mathrm{s}^{-1} \mathrm{~cm}^{-2}\left(\sim 10^{36} \mathrm{erg} \mathrm{s}^{-1}\right)$. The Cherenkov telescope HESS, working above $0.1 \mathrm{TeV}$ has not detected 1E 1740.7-2942, although significant diffuse emission has been found in the surroundings of the Galactic Center (Aharonian et al. 2006) that could cover intrinsic TeV emission from this source. It is probably premature to state that this source cannot be detected by HESS at very high energies with longer exposures, or by HESS-II in the near future.

We want to explain the emission of this object in the context of microquasars during the low hard state (Fender et al. 2003), when emission at radio and higher energies from a compact jet is produced and accretion processes are likely contributing or even dominant at X-rays. Data in the radio band and X-rays/soft gamma-rays (from RXTE and INTEGRAL) ${ }^{2}$, and upper-limits at high-energy and very high-energy gamma-rays (EGRET and HESS), have been considered. We have applied a detailed jet model to explain the data and to make predictions at high energies, and to give some constraints about the physics of the processes involved in producing the broadband emission.

\section{A jet model applied to 1E 1740.7-2942}

A model based on a freely expanding magnetized jet, in which internal energy is dominated by a cold proton plasma extracted from the accretion disk, has been developed by Bosch-Ramon et al. (2006) for microquasars in the low-hard state. The energetics of accretion, ejection, and radiation is treated consistently. We fix the jet Lorentz factor to be 1.25 , since microquasar jets during the low-hard state seem to be mildly relativistic (see, e.g., Gallo et al. 2003). We adopt a jet viewing angle of $45^{\circ}$. To keep the jet dominated by cold matter, the magnetic field energy density is taken below the equipartition value. Internal shocks occur within the jet accelerating a fraction of the jet leptons up to very high energies with a power-law energy distribution. The density of relativistic leptons is kept low to avoid their internal energy dominance, fixing the relativistic lepton to cold proton number maximum ratio to $1 / 10$. Relativistic protons are not treated here, although some amount could be present in the jet, constrained by the cold matter dominance condition (concerning their radiative properties, see e.g. Romero et al. 2005). The acceleration rate is taken $\leq 0.1 \times q_{\mathrm{e}} B c$ (see Protheroe 1999), which is balanced by electron energy losses and the accelerator size determining the maximum electron energy. The minimum energy of the non-thermal electrons has been determined equaling their gyroradius with that of protons of temperature $\sim 10^{11} \mathrm{~K}$, which dominate in our model the jet internal energy. In the context of a low mass system, as 1E 1740.7-2942 seems to be, the accelerated leptons radiate via synchrotron emission and, at higher energies, via inverse Compton (IC) scattering with synchrotron (SSC) and disk/corona photons. The disk and corona photon

1 The EGRET sensitivity in that region is confuse, as it is seen in Fig. 3 of Hartman et al. (1999), and the angular resolution is poor all along the Galactic plane.

2 CHANDRA data from the observations performed by Gallo \& Fender (2002) is not considered here due to its non-simultaneity with the observations carried out by RXTE and INTEGRAL. We note however that at that time the flux at few $\mathrm{keV}$ was about one order of magnitude lower than the flux presented here in similar energy ranges. We note that radio is either non-simultaneous, but only moderate radio flux variations have been found (Mirabel et al. 1993).
Table 1. Parameter values.

\begin{tabular}{|c|c|c|c|c|}
\hline \multicolumn{4}{|c|}{ Parameter [units] } & value \\
\hline \multicolumn{4}{|c|}{ Disk inner part temperature $[\mathrm{keV}]$} & 0.5 \\
\hline \multicolumn{4}{|c|}{ Disk luminosity $\left[\mathrm{erg} \mathrm{s}^{-1}\right]$} & $1.5 \times 10^{36}$ \\
\hline \multicolumn{4}{|c|}{ Corona photon index } & 1.6 \\
\hline \multicolumn{4}{|c|}{ Corona emission peak [keV] } & 300 \\
\hline \multicolumn{4}{|c|}{ Disk inner radius $\left[R_{\mathrm{Sch}}\right]$} & 50 \\
\hline \multicolumn{4}{|c|}{ Jet initial point (compact object $\mathrm{RF}$ ) $\left[R_{\mathrm{Sch}}\right]$} & 50 \\
\hline \multicolumn{4}{|c|}{ Jet semi-opening angle tangent } & 0.1 \\
\hline \multicolumn{4}{|c|}{ Max. ratio hot to cold lepton number } & 0.1 \\
\hline \multicolumn{4}{|c|}{ Compact object mass $\left[M_{\odot}\right]$} & 5 \\
\hline \multicolumn{4}{|c|}{ Jet Lorentz factor } & 1.25 \\
\hline \multicolumn{4}{|c|}{ Jet viewing angle $\left[{ }^{\circ}\right]$} & 45 \\
\hline$q_{\mathrm{jet} / \mathrm{accr}}$ & Accel. eff. & Diss. eff. & \multicolumn{2}{|c|}{ Prediction } \\
\hline 0.2 & 0.1 & 0.01 & \multicolumn{2}{|c|}{ up to $\mathrm{TeV}$} \\
\hline & $10^{-5}$ & 0.003 & \multicolumn{2}{|c|}{ up to $\mathrm{GeV}$} \\
\hline 0.04 & 0.1 & 0.1 & \multicolumn{2}{|c|}{ "bright" at $100 \mathrm{GeV}$} \\
\hline 0.01 & $10^{-5}$ & 0.1 & \multicolumn{2}{|c|}{ up to $100 \mathrm{MeV}$} \\
\hline
\end{tabular}

densities decrease inversely with the square of the distance when seen from the jet. However, photons coming from the disk X-ray emitting region, pretty close to the compact object, are considered as coming from behind the jet, whereas photons from the corona are treated at this stage as isotropic ${ }^{3}$. A multicolor blackbody spectrum is adopted for the disk, and a power-law plus an exponential cutoff for the corona (see Del Santo et al. 2005). Photon-photon absorption in the dominant photon fields is computed. We have also taken into account roughly the IC emission from the created pairs to compute the spectral energy distributions (SED). IC scattering and gamma-ray absorption in the low-mass star photon field are negligible. All the parameter values are summarized in Table 1. Those related to the disk and the corona radiation are taken from Del Santo et al. 2005, and those regarding the jet geometry are fixed to typical values found in the literature (e.g. Bosch-Ramon et al. 2006). We have assumed a black-hole mass of $5 M_{\odot}$.

\section{Results}

We have explored three parameters: the fraction of the accreted matter that is ejected in form of (two $)$ jets $\left(q_{\text {jet } / \text { accr }}\right)$, the acceleration efficiency, and the shock energy dissipation efficiency. For this parameter study, the electron power-law index is fixed to a typical value in Fermi I acceleration theory, 2.2. In general, a high $q_{\text {jet/accr }}$ implies low dissipation rates since otherwise it would overcome observed radio fluxes, reducing the chances of $\mathrm{TeV}$ detection but rendering still a good $\mathrm{GeV}$ emitter. A smaller $q_{\text {jet/accr }}$ allows for larger dissipation efficiencies, thus increasing the very high-energy emission. Too small $q_{\text {jet/accr }}$ does not yield radio fluxes at the observed level, let alone detectable gammaray fluxes. The explored parameter space and related features are summarized in Table 1 (bottom).

Next, we explore two representative cases: the corona dominates at X-ray energies (Del Santo et al. 2005); X-ray emission comes mainly from the jet (Markoff et al. 2001; Georganopoulos et al. 2002). The parameter values adopted in each individual case are shown in Table 2. In Fig. 1, we show computed SEDs for both cases. When the corona dominates, the jet is adopted to be mild, with high dissipation and acceleration efficiencies (to explore whether gamma-ray emission is significant).

3 The corona and the jet base, where the corona IC is more efficient, could overlap. 
Table 2. Specific parameter values for different models.

\begin{tabular}{|c|c|c|}
\hline Parameter [units] & corona & jet \\
\hline Accretion rate $\left[M_{\odot} / \mathrm{yr}\right]$ & $1.2 \times 10^{-8}$ & $2 \times 10^{-8}$ \\
\hline Corona luminosity [erg s${ }^{-1}$ ] & $3.5 \times 10^{37}$ & $10^{35}$ \\
\hline$q_{\mathrm{jet} / \mathrm{accr}}$ & 0.04 & 0.2 \\
\hline Shock dissipation efficiency & 0.1 & 0.7 \\
\hline Acceleration efficiency & 0.1 & 0.002 \\
\hline Electron power-law index & 2.2 & 1.7 \\
\hline Equipartition parameter & 0.1 & 0.3 \\
\hline
\end{tabular}

We have neglected here those components that are not relevant to model the SED of this particular source. The accretion rate, $1.2 \times 10^{-8} M_{\odot} \mathrm{yr}^{-1}$, has been fixed assuming that the total X-ray luminosity (corona+disk) is $0.05 \times c^{2}$ the total accretion rate. The corresponding Eddington accretion rate for a $5 M_{\odot}$ black hole is $2 \times 10^{-7} M_{\odot} \mathrm{yr}^{-1}$. The magnetic field at the base of the jet $^{4}$ and the (one) jet total kinetic luminosity are about $4 \times 10^{4} \mathrm{G}$ and $4 \times 10^{36} \mathrm{erg} \mathrm{s}^{-1}$, respectively. The expected fluxes are several $10^{-13} \mathrm{erg} \mathrm{cm}^{-2} \mathrm{~s}^{-1}$ at $1 \mathrm{GeV}$, and close to $10^{-13} \mathrm{erg} \mathrm{cm}^{-2} \mathrm{~s}^{-1}$ at $100 \mathrm{GeV}$. The source would have not been detected above $100 \mathrm{MeV}$, since it is below the lower EGRET limit, and its contribution to the nearby source could not be significant. HESS upper limit is at the moment above the computed emission level.

For a dominant synchrotron X-ray jet (see Fig. 1), we adopt more extreme parameter values than those explored above and neglect the corona to try to reproduce the broadband emission. The synchrotron modeling of the X-rays requires a two-sided jet with $q_{\text {jet/accr }}=0.2$, energy dissipation efficiency of $70 \%$, acceleration efficiency of 0.002 and an accretion rate taken to be a $10 \%$ of the Eddington accretion rate quoted above (i.e. $\left.2 \times 10^{-8} M_{\odot} \mathrm{yr}^{-1}\right)$. The magnetic field and jet total kinetic luminosity are $3 \times 10^{5} \mathrm{G}$ and $3 \times 10^{37} \mathrm{erg} \mathrm{s}^{-1}$. Particularly for this case, the magnetic field has been increased to reach the observed $\mathrm{X}$-ray fluxes. For the dissipation efficiency in this case, the pure radiative efficiency is very large, of about a $50 \%$. Radio fluxes are exceeded by more than one order of magnitude, and an electron power-law index of 1.7 is required. Instead of being the result of synchrotron emission, the X-rays might be produced via IC scattering. When attempting to reproduce the observed X-ray spectrum with an IC jet model with a weak corona, it is not possible to reach the X-ray fluxes through IC of external photons and/or SSC emission because the jet power requirements are too high, and it is not possible to keep the particle energy low enough as to make them radiate just up to a few hundreds of $\mathrm{keV}$ for any reasonable acceleration rate with any photon field. In addition, for the SSC model, radio constraints are also violated. We conclude, in the context of our model and after exploring a vast range of parameter values, that the corona X-ray dominated emission reproduces better the observed broadband SED than the jet X-ray dominated emission in 1E 1740.7-2942.

Finally, we have explored semi-quantitatively the radiation from the extended radio emitting jets. A magnetic field of $10^{-4} \mathrm{G}$ all along that jet region and a jet carrying at least $1 \%$ of the accreted matter was required in our model to generate radio emission up to $2-3 \mathrm{pc}$, i.e. the size of the radio lobes detected by Mirabel et al. (1992) at $8 \mathrm{kpc}$. This magnetic field is similar to what is typically found in molecular clouds (Crutcher 1999) and above the equipartition value with jet matter in those regions. We recall that 1E 1740.7-2942 could be located within a molecular cloud (Yan \& Dalgarno 1997).

4 The magnetic field decreases like $1 / z$ as far as cold matter energy density goes down along the jet.

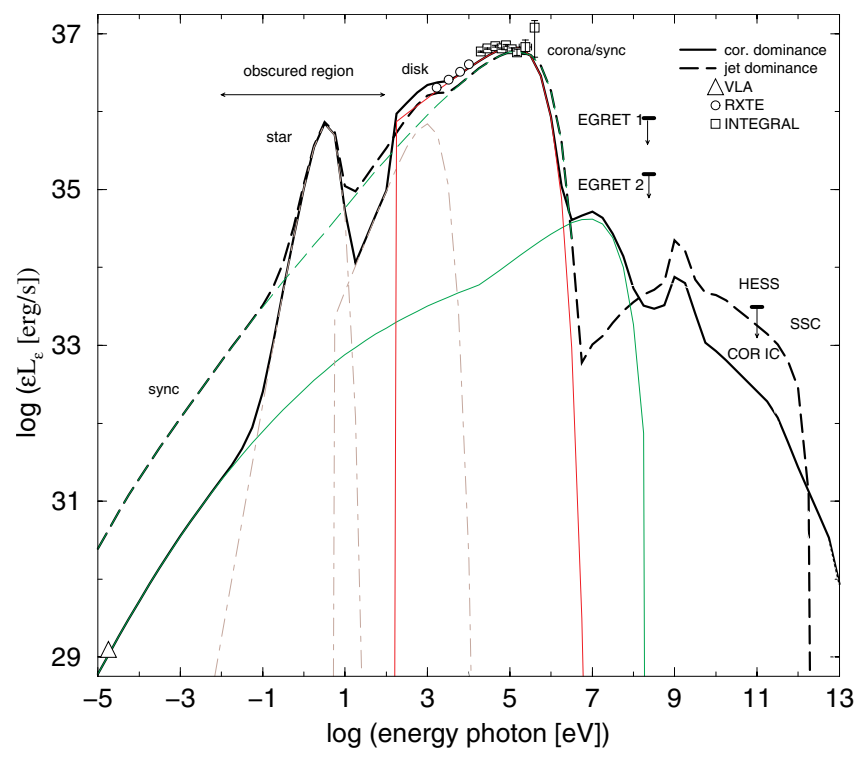

Fig. 1. Computed broad-band SED for 1E 1740.7-2942 for a dominant $\mathrm{X}$-ray corona (thick solid line) and a dominant X-ray jet (thick longdashed line) (see Table 2). Data points and observational upper-limits are also shown. Note that radio constraints are violated for the dominant $\mathrm{X}$-ray jet case. We note that dips at $\sim 100 \mathrm{MeV}$ and few $\mathrm{GeV}$ are due to photon-photon absorption in the corona and the disk photon fields respectively. At the radio band, the core luminosity at the adopted distance is shown. The slope at this range is similar to that observed (not shown explicitly; see Fender 2001, and references therein). At X-rays/soft gamma-rays, RXTE and INTEGRAL data are plotted (Del Santo et al. 2005). At high-energy gamma-rays, two upper-limits are shown: one from the spectrum of the source 3EG J1744-3011 (EGRET 1), as absolute upper-limit (Hartman et al. 1999), and the other obtained from the sensitivity limits of EGRET (EGRET 2) in the region (Hartman et al. 1999). The diffuse emission flux at $\geq 100 \mathrm{GeV}$ observed by HESS in the direction of the source is shown as an upper-limit (Aharonian et al. 2006).

\section{Discussion and summary}

There is a wide debate in the astrophysical community about the possible origin of the X-rays in microquasars since, at the present state of knowledge, the corona and the jet scenarios seem to be roughly consistent in some cases with observations (Markoff et al. 2005). In 1E 1740.7-2942, the hard X-ray emission is difficult to be explained if coming from a jet since it would imply an energetic efficiency in the jets significantly larger than for the corona emission (see Sect. 3). Actually, in the context of our model, synchrotron emission from the jet seems to be able to explain the low-hard state X-ray spectrum of the source, but it exceeds largely the observed core radio fluxes. 1E 1740.7-2942 appears to show related radio and X-ray variability (Mirabel et al. 1993), although is radio underluminous in the context of the radio-X-ray luminosity correlation found for black hole candidate X-ray binaries and associated with the accretion/ejection activity (Gallo et al. 2003; Corbel et al. 2003; Fender et al. 2003). This might be due to a particularly radiatively efficient corona. We remark that radio variations (Mirabel et al. 1993) could hardly explain a flux as large as that predicted with the synchrotron jet model (there remains the possibility that other jet models, with a much higher electron minimum energy, may be consistent with observations). All this points to the fact that, although accretion and jet phenomena are probably linked, the dominant component at X-rays can be either the jet or the disk/corona depending on the source. 
After the discovery of gamma-ray emission from microquasars (Paredes et al. 2000; Aharonian et al. 2005; Albert et al. 2006), gamma-rays are becoming of great interest as a probe for $\mathrm{X}$-ray binaries. In this context, we remark that jets in general, if sharing similar properties, are likely to emit above X-rays, reaching in some cases very high energies. Our model predicts significant amounts of gamma-rays from 1E 1740.7-2942 that might be detected by future space- and ground-based instruments like GLAST, AGILE or HESS and HESS-II, although photon-photon absorption in the disk and corona fields could prevent these detections, as can be seen comparing the two cases explored here (see Fig. 1; for more precise calculations showing the importance of the discussed effects, see e.g. Akharonian \& Vardanian 1985; Wu et al. 1993; Bednarek 1993).

Concerning the large-scale jets, Gallo \& Fender (2002) stated that the magnetic field should be $\geq 1 \mu \mathrm{G}$, in agreement with our semi-quantitative results but inferred on the basis of completely different grounds. Our estimate of the magnetic field would imply that no significant IC gamma-rays can be originated in that region, since the synchrotron emission channel would be more efficient than the IC one. It would not prevent high-energy emission to be produced via other mechanisms, like hadronic radiative mechanisms possibly at work within the mentioned molecular cloud that could surround 1E 1740.7-2942 (see, e.g., Bosch-Ramon et al. 2005).

Acknowledgements. J.M.P. and V.B.-R. acknowledge partial support by DGI of the Ministerio de Educación y Ciencia (Spain) under grant AYA-2004-07171C02-01, as well as additional support from the European Regional Development Fund (ERDF/FEDER). During this work, V.B.-R. has been supported by the DGI of the Ministerio de (Spain) under the fellowship BES-2002-2699. G.E.R. is supported by the Argentine Agencies CONICET (PIP 5375) and ANPCyT (PICT 03-13291). A.B., L.B. and M.D.S. acknowledge partial support by ASI under contract I/R/046/04.

\section{References}

Akharonian, F. A., \& Vardanian, V. V. 1985, Ap\&SS, 115, 31

Aharonian, F. A., Akhperjanian, A. G., Aye, K.-M., et al. 2005, Science, 309, 746

Aharonian F. A., Akhperjanian, A. G., Bazer-Bachi, A. R., et al. 2006, Nature, 439,695

Albert, J., Aliu, E., Anderhub, H., et al. 2006, Science, 312, 1771

Bednarek, W. 1993, A\&A, 278, 307

Bosch-Ramon, V., Aharonian, F. A., \& Paredes, J. M. 2005, A\&A, 432, 609

Bosch-Ramon, V., Romero, G. E., \& Paredes, J. M. 2006, A\&A, 447, 263

Corbel, S., Nowak, M. A., Fender, R. P., Tzioumis, A. K., \& Markoff, S. 2003, A\&A, 400, 1007

Crutcher, R. M. 1999, ApJ, 520, 706

Del Santo, M., Bazzano, A., Zdziarski, A. A., et al. 2005, A\&A, 433, 613

Dickey, J. M., \& Lockman, F. J. 1990, ARA\&A, 28, 215

Fender, R. P. 2001, MNRAS, 322, 31

Fender, R. P., Gallo, E., \& Jonker, P. G. 2003, MNRAS, 343, L99

Gallo, E., \& Fender, R. P. 2002, MNRAS, 337, 869

Gallo, E., Fender, R. P., \& Pooley, G. G. 2003, MNRAS, 344, 60

Georganopoulos, M., Aharonian, F. A., \& Kirk, J. G. 2002, A\&A, 388, 25

Hartman, R. C., Bertsch, D. L., Bloom, S. D. et al. 1999, ApJ, 123, 79

Hertz, P., \& Grindlay, J. E. 1984, ApJ, 278, 137

Markoff, S., Falcke, H., \& Fender, R. 2001, A\&A, 372, L25

Markoff, S., Nowak, M. A., \& Wilms, J. 2005, ApJ, 635, 1203

Martí, J., Mirabel, I. F., Chaty, S., \& Rodríguez, L. F. 2000, A\&A, 363, 184

Mirabel, I. F., Rodríguez, L. F., Cordier, B., Paul, J., \& Lebrun, F. 1992, Nature, 358,215

Mirabel, I. F., Rodriguez, L. F., Cordier, B., Paul, J., \& Lebrun, F. 1993, A\&AS, 97, 193

Paredes, J. M., Martí, J., Ribó, M., \& Massi, M. 2000, Science, 288, 2340

Protheroe R. J. 1999, in Topics in Cosmic-Ray Astrophysics, 247 [arXiv: astro-ph/9812055]

Romero, G. E., Christiansen, H., \& Orellana, M. 2005, ApJ, 632, 1093

Smith D. M., Heindl W. A., \& Swank J. H. 2002, ApJ, 578, 129

Sunyaev, R., Churazov, E., Gilfanov, M., et al. 1991, ApJ, 383, L49

Wu, M., Zhang, C., \& Li, T. 1993, Ap\&SS, 204, 141

Yan, M., \& Dalgarno, A. 1997, ApJ, 481, 296 